Faculty of Science

Faculty Publications

This is a post-review version of the following article:

Dynamic and Coverage Effects in EIS

D. A. Harrington

2013

The final published version of this article can be found at:

http://dx.doi.org/10.1149/04513.0003ecst

Citation for this paper:

Harrington, D.A. (2013). Dynamic and Coverage Effects in EIS. ECS Transactions, 45(13), 3-14. http://dx.doi.org/10.1149/04513.0003ecst 
Accepted version of ECS Transactions 45 (2013) 3-14. doi: 10.1149/04513.0003ecst

(C) The Electrochemical Society, Inc. 2013. All rights reserved. Except as provided under U.S. copyright law, this work may not be reproduced, resold, distributed, or modified without the express permission of The Electrochemical Society (ECS).

\title{
Dynamic and Coverage Effects in EIS
}

\author{
David. A. Harrington \\ Department of Chemistry, University of Victoria, Victoria, BC, V8W 3V6, Canada
}

The effect of sweep rate on the validity of impedance measurements made dynamically when sweeping the potential is investigated by numerical solution of the differential equations for a simple adsorption reaction. The same method is used to show that dynamic EIS can detect a nucleation-growth-collision mechanism through a negative time constant, and that measurement in the unstable regime is possible, even though the data is not Kramers-Kronig transformable. The utility of the concept of the rate-determining step in EIS is demonstrated for a simple example.

\section{Introduction}

Electrochemical Impedance Spectroscopy (EIS) is well accepted as a useful technique, but its use is still dogged with some questions of interpretation and validity. I address here three of these questions. Two of them arise in the case of dynamic electrochemical impedance spectroscopy (dEIS), where the "dc" potential is slowly changed, usually in a potential sweep experiment: (1) How fast can one sweep before the measured impedance is invalid? (2) Can impedance of unstable systems (which violate the Kramers-Kronig relationships) be sensibly measured? These questions are investigated theoretically by directly solving the relevant differential equations for simple systems involving adsorption. The equations are solved directly for the ac $+\mathrm{dc}$ potential waveform, without any assumptions or approximations about separability of the ac and dc parts.

A third question relates to the ability to detect an adsorbed species by EIS. Each additional adsorbed species typically generates an additional semicircle in a Nyquist plot, but it is evident that if these additional semicircles are small enough that for practical purposes they cannot be detected, then the apparent number of adsorbed species will be less than the actual number. Under what circumstances will this happen? I show that introduction of the idea of the rate-determining step (rds) into EIS provides a useful framework for understanding this issue. The specific case of a two-step reaction mechanism with a single adsorbed species is considered, and it is shown that when the first or second steps are rate determining, the two semicircles found for this mechanism collapse down to one, for reasons that are intuitively easy to understand.

\section{Results and Discussion}

\section{Effect of Sweep Rate in Dynamic EIS}

I address the effect of sweep rate for the case of a simple adsorption reaction obeying Langmuir kinetics (Eqs. [1] and [2]), where the symbols have their usual meaning, and the rate $v_{1}$ and rate constant $k^{0}$ have units $\mathrm{mol} \mathrm{m}^{-2} \mathrm{~s}^{-1}$. Mass transport of $\mathrm{A}^{-}$is fast and its 
concentration has been included in $k^{0}$. The differential equation [3], where $\Gamma_{\mathrm{m}}$ is the concentration of sites on the clean surface in mol $\mathrm{m}^{-2}$, was solved for the ac + sweep potential program [4]. Unless specified otherwise, a $5 \mathrm{mV} / \mathrm{s}$ sweep with a $5 \mathrm{mV} \mathrm{rms} 2 \mathrm{~Hz}$ ac signal was used. The solution was obtained at a density of 192 points per second, using Maple $^{\mathrm{TM}}$ 's Rosenbrock stiff solver requiring absolute and relative errors of better than $10^{-6}$. The coverage as a function of time so obtained was then used to calculate the faradaic current density using Eq. [5].

$$
\begin{aligned}
& \mathrm{M}(\text { site })+\mathrm{A}^{-}(\mathrm{aq}) \rightleftarrows \mathrm{MA}(\mathrm{ads})+\mathrm{e}^{-} \\
& v_{1}=k^{\mathrm{o}}(1-\theta) \exp \left(\left(1-\beta_{1}\right) F\left(E-E^{\mathrm{o}}\right) / R T\right)-k^{\mathrm{o}} \theta \exp \left(-\beta_{1} F\left(E-E^{\mathrm{o}}\right) / R T\right) \\
& \Gamma_{\mathrm{m}} \mathrm{d} \theta / \mathrm{d} t=v_{1} \\
& E=E_{\mathrm{i}}+v t+a \sin (2 \pi f t) \\
& j_{\mathrm{f}}=F v_{1}
\end{aligned}
$$

The output of this synthetic "experiment" was analyzed by the same software that is used to analyze the real dynamic EIS data (1). At the "sampling" frequency of $192 \mathrm{~Hz}$, successive samples of the current density $j_{\mathrm{f}}$, the ac potential $a \sin (2 \pi f t)$ and the "dc" potential $E_{\mathrm{i}}+v t$ were used, e.g., every third current density produced by the solver was used. The analysis program performs a baseline subtraction as described in (1) and then carries out 32 point fast fourier transforms $(0.5 \mathrm{~s}$ long) on the current density and ac potential centered every $2.5 \mathrm{mV}$ along the voltammogram. The faradaic impedance is then calculated by dividing the complex number for potential by the complex number for current in the usual way. A correction is applied for the non-simultaneous sampling of current and potential.

The theoretical faradaic impedance of this reaction is a series combination of a charge-transfer resistance and pseudocapacitance, given by Eqs. [6] and [7] (2). The accuracy of the method was assessed by a test at zero sweep rate at $E=E^{\mathrm{o}}(\theta=0.5), k^{0}=$ $2.5 \times 10^{-10} \mathrm{~mol} \mathrm{~cm}^{-2} \mathrm{~s}^{-1}, \Gamma_{\mathrm{m}}=2.5 \times 10^{-9} \mathrm{~mol} \mathrm{~cm}^{-2}, \beta_{1}=0.5$, for which the theoretical values are $R_{\mathrm{ct}}=2.1303 \mathrm{k} \Omega \mathrm{cm}^{2}$ and $C=2.3471 \mathrm{mF} \mathrm{cm}^{-2}$. The fourier transformed data from the "experiment" gave an impedance at $2 \mathrm{~Hz}$ from which the calculated values were $R_{\mathrm{ct}}=2.1252 \mathrm{k} \Omega \mathrm{cm}^{2}$ and $C=2.3474 \mathrm{mF} \mathrm{cm}^{-2}$. The discrepancy may come from errors in the differential equation solver, in the fourier analysis, or in the theory (which strictly only applies to infinitely small perturbation signals), but the accuracy exceeds that obtainable in most experimental situations and is certainly sufficient to test out dynamic effects.

$$
\begin{gathered}
R_{\mathrm{ct}}^{-1}=F\left(\partial v_{1} / \partial E\right)_{\theta} \\
C=-\Gamma_{\mathrm{m}} F\left(\partial v_{1} / \partial E\right)_{\theta} /\left(\partial v_{1} / \partial \theta\right)_{E}
\end{gathered}
$$

Fig. 1 shows the effect of sweep rate on the voltammograms and calculated circuit elements for a fixed rate constant. As expected, increasing the sweep rate makes the voltammograms more irreversible, and the peak potentials shift to more positive values. At $1 \mathrm{mV} / \mathrm{s}$, the $R_{\mathrm{ct}}$ and $C$ values from the solution of the differential equation are very close to those calculated from the theoretical expressions in Eqs. [6] and [7] (Fig. 1, top right). The theory, as usual, assumes quasi-steady state and a small perturbation ac signal. At $5 \mathrm{mV} / \mathrm{s}$, there is a systematic error between theory and the solution of the differential equation, for example the difference between the two capacitances at the peak is about $7 \%$. The lack of noise suggests that this is a real dynamic effect rather than an artifact of 

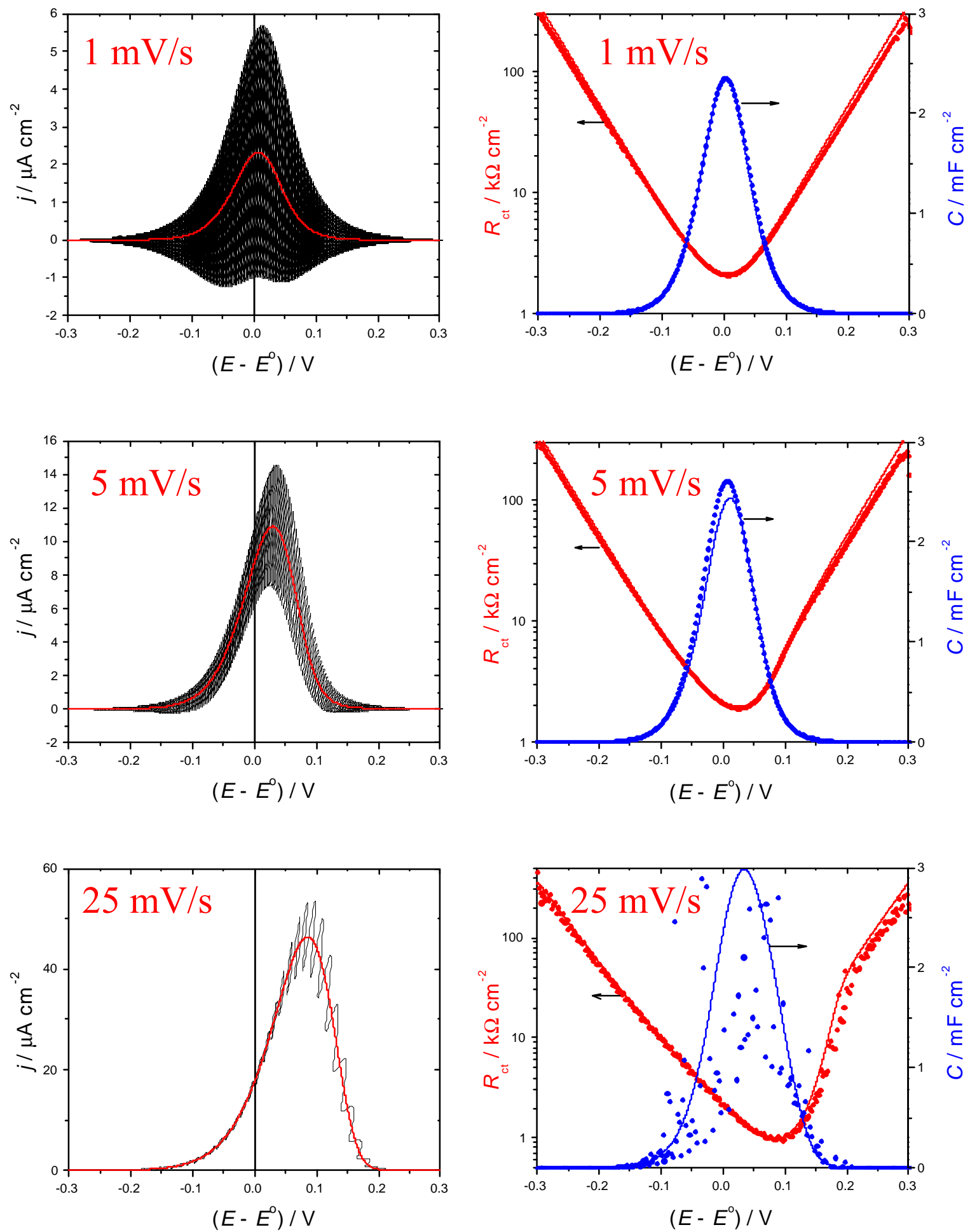

Figure 1. Effect of sweep rate on dEIS. Left panels show voltammograms for the simple adsorption reaction [1], with $k^{0}=2.5 \times 10^{-10} \mathrm{~mol} \mathrm{~cm}^{-2} \mathrm{~s}^{-1}, \Gamma_{\mathrm{m}}=2.5 \times 10^{-9} \mathrm{~mol} \mathrm{~cm}^{-2}, \beta_{1}=$ 0.5 . The red line is the current density without any ac potential perturbation, the black line is the rapidly changing current density when there is an ac potential perturbation of $5 \mathrm{mV}$ rms at $2 \mathrm{~Hz}$. Right panels show the charge transfer resistance (red) and capacitance (blue) from the steady-state small signal theory (Eqs. [6] and [7], solid line) and from numerical solution of the differential equation and fourier analysis (points). 
$k^{0}=250 \mathrm{pmol} \mathrm{cm}^{-2} \mathrm{~s}^{-1}$
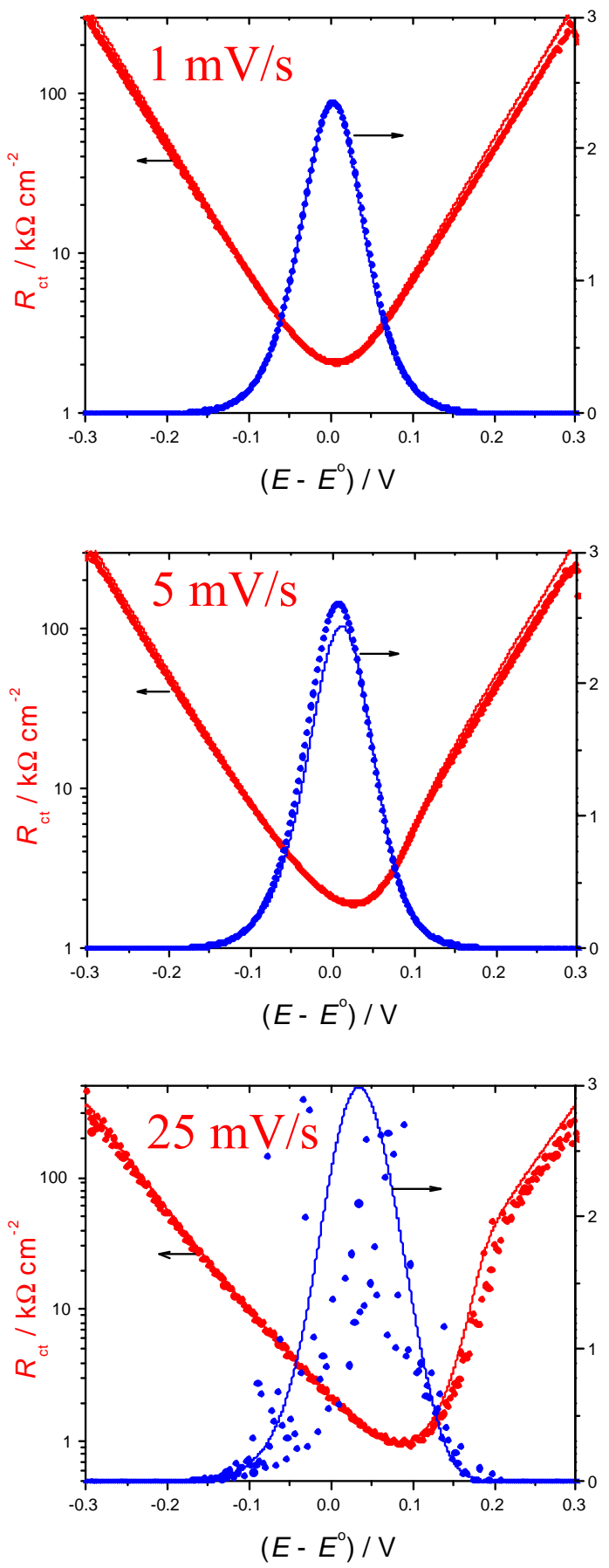

$k^{\mathrm{o}}=1250 \mathrm{pmol} \mathrm{cm}^{-2} \mathrm{~s}^{-1}$
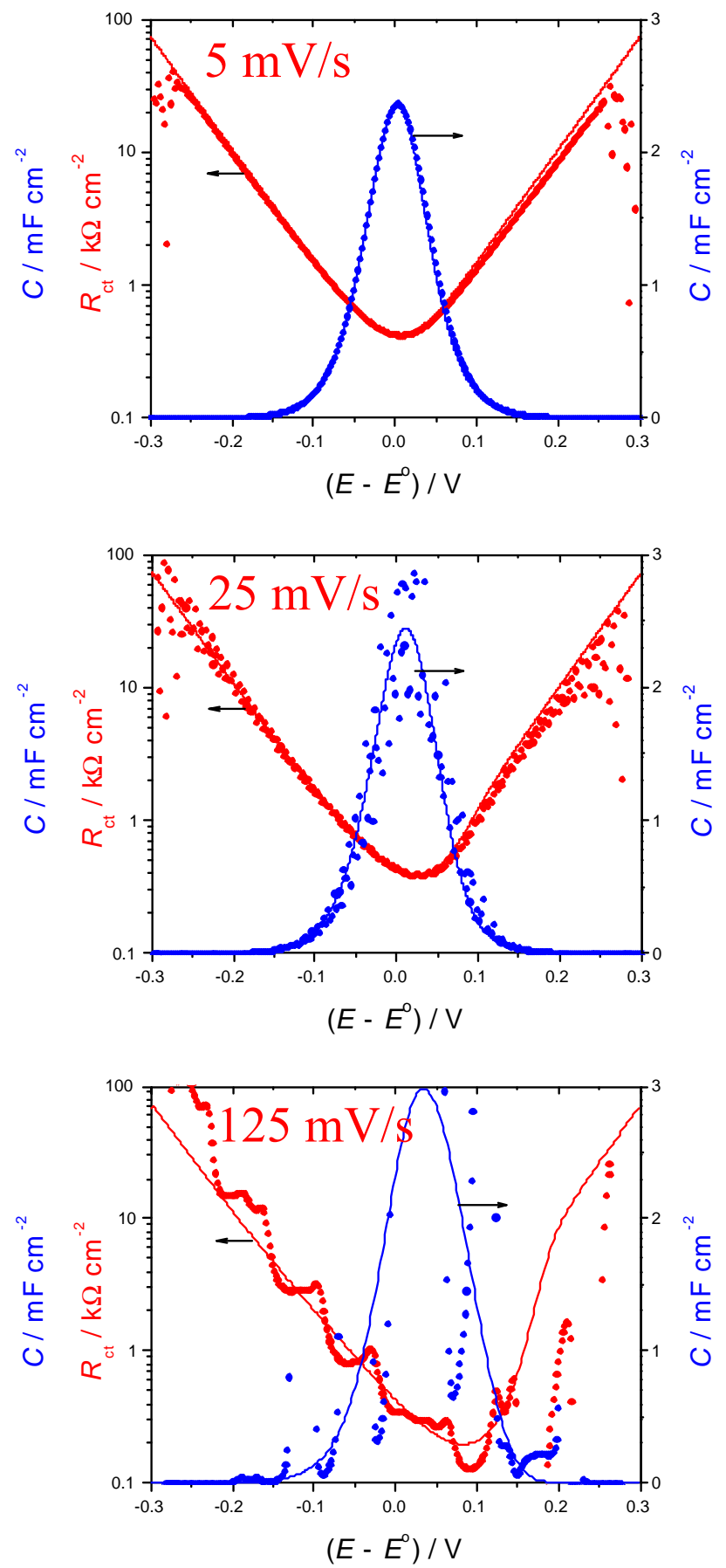

Figure 2. Effect of rate constant on dEIS. Left panels show $R_{\mathrm{ct}}$ (red) and $C$ (blue) for the simple adsorption reaction [1], with $k^{0}=2.5 \times 10^{-10} \mathrm{~mol} \mathrm{~cm}^{-2} \mathrm{~s}^{-1}, \Gamma_{\mathrm{m}}=2.5 \times 10^{-9} \mathrm{~mol} \mathrm{~cm}^{-2}$, $\beta_{1}=0.5$ and with an ac potential perturbation of $5 \mathrm{mV} \mathrm{rms}$ at $2 \mathrm{~Hz}$. The solid line is from the steady-state small signal theory (Eqs. [6] and [7]) and points are from numerical solution of the differential equation and fourier analysis. The right panels show data at five times the sweep rate and five times the rate constant. 
the sampling rate, baseline correction or other effects of the analysis procedure. By $25 \mathrm{mV} / \mathrm{s}$, the "measured" capacitance has serious errors, to the extent that extraction of parameters such as $k^{\circ} / \Gamma_{\mathrm{m}}$ or $\beta_{1}$ from this data would likely be unreliable, though the resistance shows some residual coherence.

Fig. 2 shows the effect of rate constant on the resistance and capacitance values at some selected sweep rates. The right panels are at five times the sweep rate and five times the rate constant. This leaves the theoretical capacitances unchanged, and the resistances decrease by a factor of five. For the top panels, it is evidence that an increase in the rate constant by a factor of five allows the sweep rate to be increased by a factor of five without much degradation in the "measured" data. Even at $25 \mathrm{mV} / \mathrm{s}$, the data is reasonable for the higher rate constant, albeit somewhat noisy, when it was not for the lower rate constant at that same sweep rate. However, there is a limit to the ability of an increased rate constant to allow faster sweep rates as shown in the bottom panels, where an increase in rate constant by five leads only to a meaningless result at five times the sweep rate.

These results may be discussed in the context of literature estimates of how fast one may sweep and still measure valid impedances. There are some who maintain that "impedance ... is only defined in the steady state; impedance has no meaning in time dependent systems..." (3). Although this may be strictly true, it is intuitively evident that if the system changes slowly enough that the key variables describing the system (potential, coverages, concentrations) do not significantly change in the time to measure the impedance at a single frequency (typically one or two cycles), then EIS measurements can be made. Indeed, ac voltammetry was used in the early days of electroanalytical chemistry, without much quantitative consideration as to how fast one could sweep while applying a perturbation at a single frequency, though Underkofler and Shain noted that for small signals the angular frequency $\omega$ should be related to the sweep rate $v$ by

$$
\omega>>v / \Delta E
$$

where $\Delta E$ is the amplitude. This was deduced in order to make the transient term in their theory small, but is essentially a statement that the change in sweep potential during an ac cycle must be small compared to the amplitude. Smith's pioneering work on how to rapidly measure impedances led to the multisine method widely used today, but the only discussion of this issue is a single paper (5), in which digital simulation was used to show that frequencies as low as $15.6 \mathrm{~Hz}$ (amplitude $3.5 \mathrm{mV} \mathrm{rms}$ ) could be used at $50 \mathrm{mV} / \mathrm{s}$ for a reversible solution redox process. This amounts to a change of $3.2 \mathrm{mV}$ in one ac cycle, a surprisingly large change, but one in which the left-hand side of Eq. [8] is ten times the right if $\Delta E$ is interpreted as the peak amplitude ( $\sqrt{2}$ times the rms amplitude). Later I analysed a surface mechanism (6), and conjectured that for such mechanisms

$$
\omega>v(F / R T)
$$

which led to a similar conclusion that about $1 \mathrm{~Hz}$ at $5 \mathrm{mV} / \mathrm{s}$ is acceptable. Here the idea is a little different, that the sweep potential in a cycle should not change by much compared to the thermal potential, $R T / F$, which defines a "small" potential for electrochemical processes. 
Garland, Petit and Roy (7) reiterated that Eq. [8] should hold (though they used rms amplitude), but they also pointed out that just as the sweep potential should not change much during an ac period, neither should the current, and suggested the additional constraint

$$
\omega>>(\mathrm{d} j / \mathrm{d} t) / \Delta j_{\mathrm{rms}}
$$

where $\Delta j_{\mathrm{rms}}$ is the rms amplitude of the ac current density response at the fundamental angular frequency $\omega$. I propose that in similar vein, the coverages, concentrations and other system parameters also must not change too much in an ac period, and therefore that in the present adsorption case

$$
\omega>>(\mathrm{d} \theta / \mathrm{d} t) / \Delta \theta_{\mathrm{rms}}
$$

The difficulty with this relationship is of course that the rms amplitude of the coverage perturbation, $\Delta \theta_{\text {rms }}$, is not typically known a priori, though it may be estimated once a model has been assumed and fitted.

A full quantitative discussion of the present results against these criteria will be given elsewhere, but some preliminary analysis may be readily made. Firstly, there is already some significant error for $5 \mathrm{mV} / \mathrm{s}$ and $2 \mathrm{~Hz}$ in Fig. 1, even though conditions [8] and [9] are satisfied. Conversely, some sensible though noisy results may be obtained even at $25 \mathrm{mV} / \mathrm{s}$ and $2 \mathrm{~Hz}$ where the left-hand side of [8] is only four times the right-hand side. Secondly, the maximum sweep rate at which valid results are obtained is dependent on the rate constant, and so cannot be solely described by either [8] or [9]. Looking at the voltammograms with and without the ac at different sweep rates (Fig. 1, left panels), it seems that the current is changing most in an ac cycle at $25 \mathrm{mV} / \mathrm{s}$ (bottom left). Since the dc voltammetry currents scale approximately with sweep rate, but the peak widths are approximately independent of sweep rate, $(\mathrm{d} j / \mathrm{d} t)$ will increase with sweep rate. On the other hand, the figure shows that the ac currents have a weak dependence on sweep rate, so that the rhs of Eq. [10] will increase with sweep rate, and Eq. [10] should eventually constrain the validity.

One might also anticipate that if the amplitude of the ac current density is large compared to the de current density, the small signal analysis implicit in the impedance theory fails and agreement is no longer obtained between the theory and the differential equation solution. However, Fig. 1 shows that in the $1 \mathrm{mV} / \mathrm{s}$ case, where the best agreement is obtained, the ac current density is the largest fraction of the dc current density, and is actually comparable to it. Evidently, the measured impedance is insensitive to a significant departure from the perturbation regime.

From the parameter space explored here, it appears as though frequencies as low as 2 $\mathrm{Hz}$ at $5 \mathrm{mV} / \mathrm{s}$ give good accuracy, but worse results may be anticipated at lower rate constants than about $2.5 \times 10^{-10} \mathrm{~mol} \mathrm{~cm}^{-2} \mathrm{~s}^{-1}$.

Can Unstable Systems be Measured in Dynamic EIS?

Stability is a requirement for the Kramers-Kronig relationships to hold (8), and violation of these conditions for experimental data is often taken to be synonymous with 
an indication of bad data. Instability is often assumed to mean the same as "drift" in the sense that the system changes over the course of the measurement of an impedance spectrum. However, the derivation of the Kramers-Kronig (KK) relationships makes it clear that stability is meant in the technical sense of the absence of poles of the transfer function in the left half of the complex frequency " $s$ " plane. In the time domain this means that a small fluctuation is followed by relaxation back to steady-state (positive system time constant), whereas an unstable system shows divergence away from steady state after a fluctuation (negative system time constant).

The above sweep rate effects are more akin to system drift effects as the time constant $\tau=R_{\mathrm{ct}} C$, which is a system time constant for the interfacial admittance, is always positive. Nucleation-growth-collision (NGC) of islands of an adsorbed species growing on a surface provides a simple example of a system that is unstable, and shows a negative time constant when the islands are growing without significant collisions. We suggested that a negative time constant observed in the experimental dEIS results for methanol oxidation on platinum was an indication of growth of islands of $\mathrm{OH}(\mathrm{ads})$ in a sea of $\mathrm{CO}(\mathrm{ads})$ via an NGC mechanism (9). However, the fact that this data may not be KK transformable raises questions about whether this conclusion about NGC growth is valid, and if rate parameters could be extracted reliably from the measured spectra.

To address these questions, the same methodology as above is used to solve the differential equation for a simple irreversible instantaneous NGC process. The rate law [2] is replaced by the rate law [12], where the total island perimeter $l$ per unit area of electrode surface (units: $\mathrm{m} / \mathrm{m}^{2}=\mathrm{m}^{-1}$ ) is related to the coverage by Eq. [13], where $\Omega\left(\mathrm{m}^{-2}\right.$ ) is the nucleation density.

$$
\begin{gathered}
v_{1}=k^{\mathrm{o}} \exp \left(\left(1-\beta_{1}\right) F\left(E-E^{\mathrm{o}}\right) / R T\right) l \\
\\
l=2(1-\theta)[-\Omega \pi \ln (1-\theta)]^{1 / 2}
\end{gathered}
$$
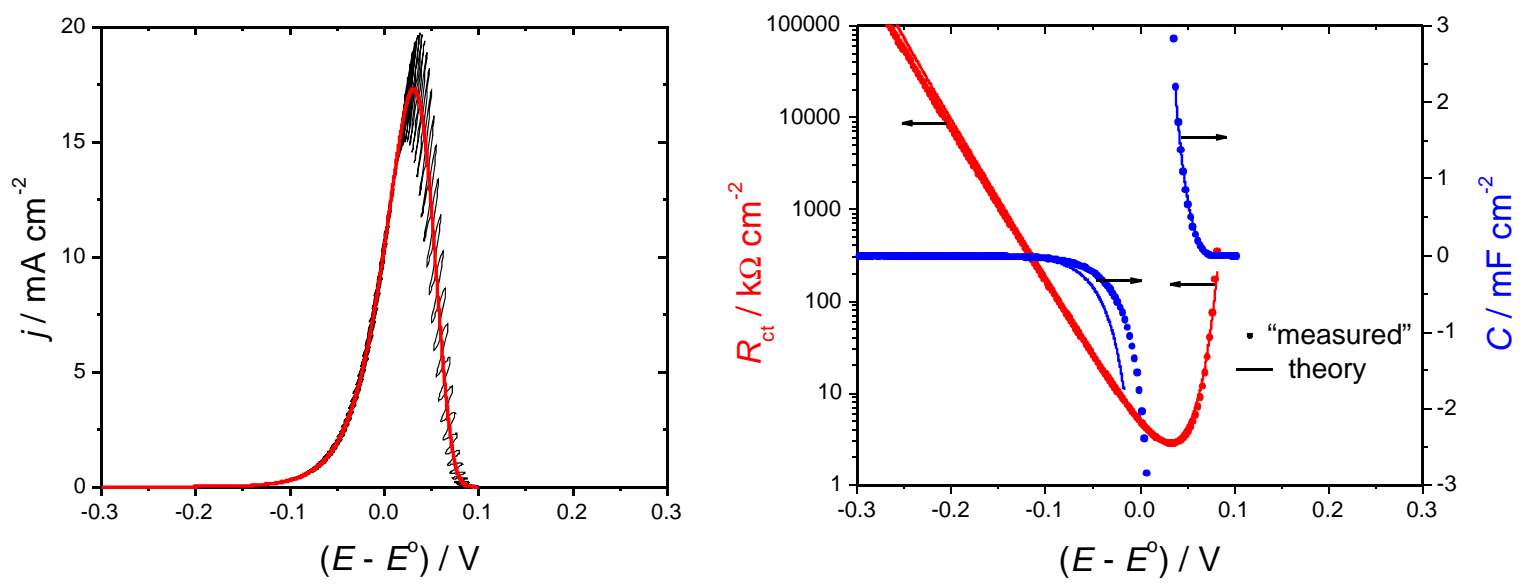

Figure 3. Voltammogram, $R_{\text {ct }}$ and $C$ for an irreversible instantaneous NGC adsorption reaction. Left: Voltammogram. Red line is without an ac perturbation, black line is with a $5 \mathrm{mV}$ rms $2 \mathrm{~Hz}$ ac potential applied. Right: $R_{\mathrm{ct}}$ (red) and $C$ (blue) The solid line is from the steady-state small signal theory (Eqs. [6] and [7]), and points are from numerical solution of the differential equation and fourier analysis. Parameters in Eqs. [12] and [13]: $k^{0} \Omega^{1 / 2}=7.5 \times 10^{-12} \mathrm{~mol} \mathrm{~cm}^{-2} \mathrm{~s}^{-1}, \Gamma_{\mathrm{m}}=2.5 \times 10^{-9} \mathrm{~mol} \mathrm{~cm}^{-2}, \beta_{1}=0.5$. Sweep rate 5 $\mathrm{mV} / \mathrm{s}$. 
The equivalent circuit is the same as before, with $R_{\mathrm{ct}}$ and $C$ calculated from Eqs. [6] and [7]. Fig. 3 shows a voltammogram and corresponding $R_{\mathrm{ct}}$ and $C$, both from theory and solution of the differential equation.

The charge-transfer resistance calculated from Eq. [6] is positive at all potentials, but the capacitance calculated from Eq. [7] is negative for low coverages and positive for higher coverages. At low coverages, adsorption causes an increase in perimeter and hence in rate, so a fluctuation that increases the coverage leads to further increase the coverage in an unstable positive feedback process, associated with a negative time constant $R_{\mathrm{ct}} C$ with $R_{\text {ct }}$ positive and $C$ negative. At higher coverages, the perimeter decreases as the coverage increases, and so a fluctuation that increases coverage would lead to a coverage decrease, i.e., the behavior is stable again. Solution of the differential equation shows that the impedance that would be measured in the dEIS experiment shows the expected negative capacitance behavior at low coverages (potentials) and positive capacitance at higher coverages (potentials), though there is a significant difference between the theoretical and measured values. Experiments with other parameters showed that this error in capacitance persisted, even though the simultaneously measured resistance was accurate.

The conclusion is that dEIS can successfully measure an unstable process, despite the fact that the impedance is not KK transformable. The negative time constant can be used as evidence for a possible NGC process, though the parameters extracted may be inaccurate. The ability to measure an unstable process by dEIS should not be surprising, since unstable NGC peaks are routinely measured in cyclic voltammetry. This situation is different from steady-state EIS, where the steady state for an unstable process cannot be maintained. However, the unstable region in a voltammetry experiment persists only for a short time before the behavior becomes stable again; the deterministic evolution of the system in the unstable regime can be measured for at least some ac perturbations and system parameters.

\section{The Rate-Determining Step in EIS}

The concept of the rate-determining step is widely used in chemical kinetics, and its use in interpreting Tafel slopes is well known in electrochemistry, but it has been little used in EIS. One reason for this is perhaps that the linearization of the rate equations in the derivation of EIS spectra leads to tractable equations even for complicated multistep reaction mechanisms, and therefore the simplification brought about by the concept of the rds is unnecessary. Another reason might be that the exponential potential dependence of the rate constants for charge-transfer steps means that the step that is rate determining at one potential may not be at another potential, making the concept less useful. However, we illustrate here that the reasons for semicircles in the Nyquist plot "disappearing" can be rationalized easily by introducing the concept of the rds. The similar idea of "fast" and "slow" species was recently introduced by Sengoku et al. as a simplifying concept (10).

The simplifying role of the rds in EIS is illustrated by a two step adsorption reaction mechanism, Eqs. [14] and [15], with governing equations [16] - [19]. 
(a) General case

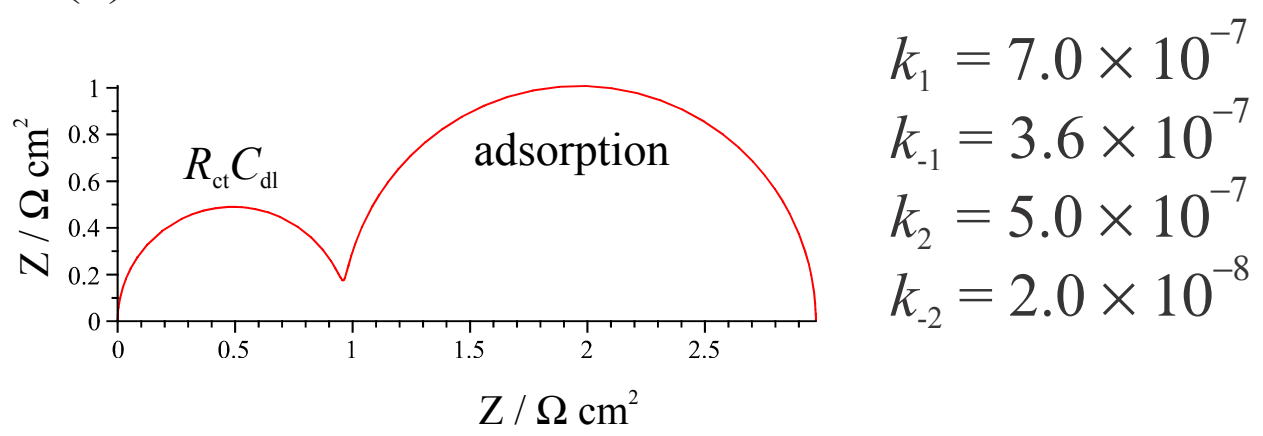

(b) Step $1 \mathrm{rds}$

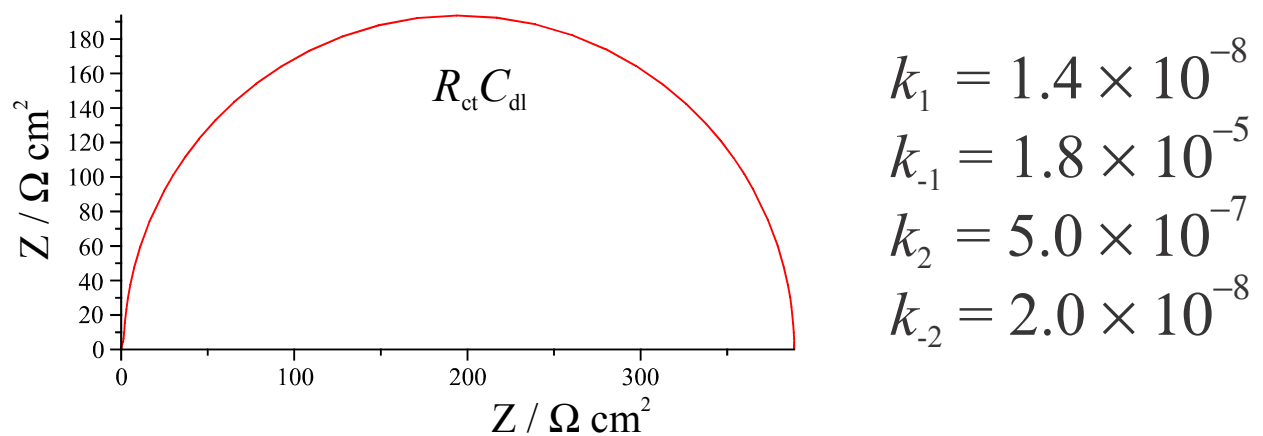

(c) Step 2 rds

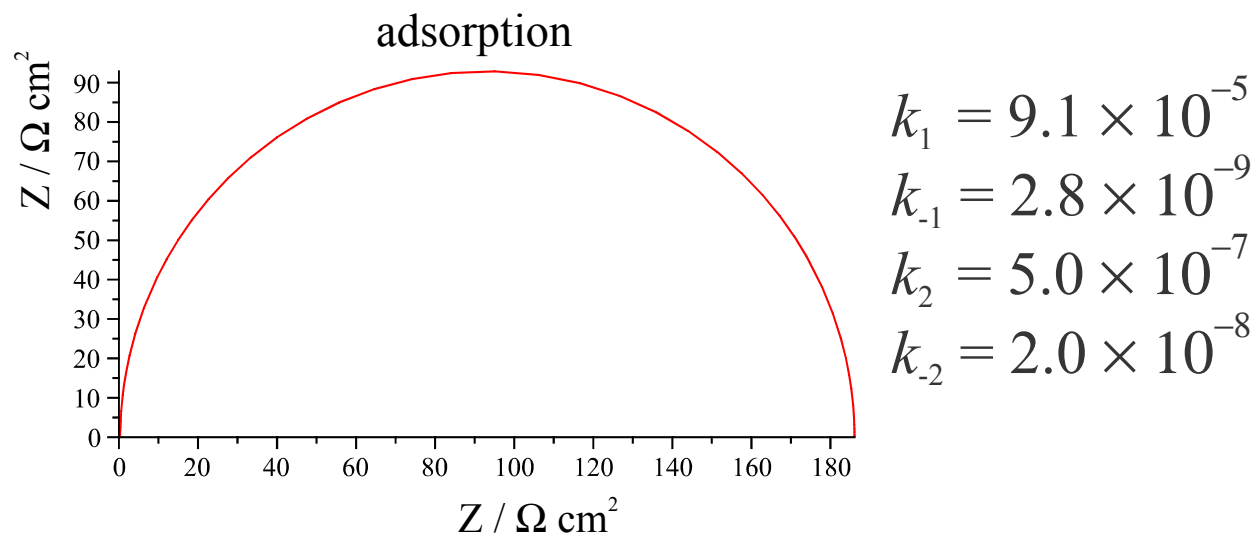

Figure 4. EIS spectra for the two-step mechanism of Eqs. [14] and [15]. Rate constants

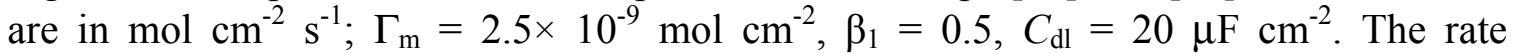
constants are chosen to satisfy the thermodynamic constraint $k_{1}{ }^{\mathrm{eq}} k_{2} / k_{-1}{ }^{\mathrm{eq}} k_{-2}=1$, and correspond to different overpotentials with the same values of $k_{1}{ }^{\mathrm{eq}}, k_{-1}{ }^{\mathrm{eq}}, k_{2}$, and $k_{-2}$. 


$$
\begin{aligned}
& \mathrm{M}(\text { site })+\mathrm{A}^{-}(\mathrm{aq}) \rightleftarrows \mathrm{MA}(\mathrm{ads})+\mathrm{e}^{-} \\
& \mathrm{MA}(\mathrm{ads}) \rightleftarrows \mathrm{M}(\text { site })+\mathrm{A}(\mathrm{aq}) \\
& \text { (step 1) } \\
& \text { (step 2) } \\
& v_{1}=k_{1}(1-\theta)-k_{-1} \theta=k_{1}{ }^{\mathrm{eq}}(1-\theta) \exp \left(\left(1-\beta_{1}\right) F \eta / R T\right)-k_{-1}{ }^{\mathrm{eq}} \theta \exp \left(-\beta_{1} F \eta / R T\right) \\
& v_{2}=k_{2} \theta-k_{-2}(1-\theta) \\
& \Gamma_{\mathrm{m}} \mathrm{d} \theta / \mathrm{d} t=v_{1}-v_{2} \\
& j_{\mathrm{f}}=F v_{1}
\end{aligned}
$$

The impedance for this mechanism can be derived using standard methods, e.g., (2), without any assumptions as to which step is the rds. The result is that this mechanism shows two semicircles in a Nyquist plot, a high-frequency semicircle associated with the charge-transfer resistance and double layer capacitance, and a low-frequency semicircle associated with relaxation of the adsorbed species (Fig. 4(a)).

Suppose now that we make the simplifying assumption that step 1 is slow and therefore rate determining. That is, we take $k_{2}>>k_{1}$. In this circumstance the removal of the adsorbed species by step 2 occurs immediately it is formed ${ }^{1}$. This means that the coverage is kept low, and the rate of step 2 must follow the rate of step 1, i.e., the steadystate approximation holds (Eq. [18] is set to zero), and therefore $v_{2}=v_{1}$. The current density is then found as in Eq. [20].

$$
j_{\mathrm{f}}=F v_{1}=F k_{1}(1-\theta) \approx F k_{1}
$$

Oscillation of the potential leads to oscillation of $k_{1}$ and thence to oscillation of $j_{\mathrm{f}}$ without a phase shift. Therefore the faradaic impedance reduces to a resistance, and the interfacial impedance should show a single semicircle. Evaluation of the general equations for the case $k_{2}>>k_{1}$ verifies this prediction, as shown in Fig. 4(b). We see only the high frequency semicircle associated with the charge-transfer and double layer. The low frequency semicircle associated with relaxation of the surface coverage has become very small, because there is negligible coverage of adsorbed species.

The other extreme is for step 2 to be slow and rate determining. As usual, the faster steps prior to the rds are considered as pre-equilibria. Here we take $k_{1}, k_{-1}>>k_{2}$ and step 1 has time to come to equilibrium as the adsorbed species "queue up" for step 2. (Actually we only require the sum $k_{1}+k_{-1}$ to be much greater that $k_{2}$.) Then coverage is determined by the equilibrium expression [21], which solves to give [22].

$$
\begin{gathered}
K_{1}=k_{1} / k_{-1}=\left(k_{1}{ }^{\mathrm{eq}} / k_{-1}{ }^{\mathrm{eq}}\right) \exp (F \eta / R T)=\theta /(1-\theta) \\
\theta=K_{1} /\left(K_{1}+1\right)
\end{gathered}
$$

Oscillation of the potential leads to oscillation of $K_{1}$ and therefore to oscillation of $\theta$ without a phase shift. The current density is then found from [18] and [19] to be

$$
j_{\mathrm{f}}=F v_{1}=F\left(v_{2}+\Gamma_{\mathrm{m}} \mathrm{d} \theta / \mathrm{d} t\right)=F k_{2} \theta+F \Gamma_{\mathrm{m}} \mathrm{d} \theta / \mathrm{d} t
$$

The first term will give a contribution to the current which is in phase with the coverage, which in turn is in phase with the potential, i.e., the behavior is resistive. The

\footnotetext{
${ }^{1}$ The back reaction of step 1 can also serve as the fast removal. Strictly we only require $k_{2}+k_{-1}>>k_{1}$ but we typically assume $k_{-1} \approx 0$.
} 
second term will give a contribution which is $180^{\circ}$ out of phase (capacitive), because of the presence of the derivative $\mathrm{d} \theta / \mathrm{d} t$ (i $\omega \Delta \theta$ in the frequency domain). The faradaic admittance is therefore a parallel combination of a resistance and capacitance. Addition of the double layer capacitance in parallel alters only value of the capacitance, and so a single $R C$ semicircle should be seen in a Nyquist plot. Evaluation of the general equations for the case $k_{1}+k_{-1}>k_{2}$ verifies this prediction, as shown in Fig. 4(c). This semicircle is associated with the coverage relaxation. Taking the first step as a rapid equilibrium ensures that coverage changes instantaneously with the potential, and we never find the charge-transfer regime where the electron-transfer rate changes without a coverage change: the high-frequency $R_{\mathrm{ct}} C_{\mathrm{dl}}$ semicircle has disappeared. This is emphasized by the fact that the observed resistance comes from the first term in [23], which is associated with the step without electron transfer.

The single semicircle behaviors associated with steps 1 or 2 being the rds are of course the extremes of a continuum in which the two semicircles dominate to different extents.

\section{Conclusions}

The validity of dEIS and the conditions under which artifacts can arise may be investigated by numerically solving the differential equations for the exact $\mathrm{dc}+\mathrm{ac}$ potential waveform applied. The results are independent of the usual assumptions made, e.g., the separability of the ac and dc parts, the small amplitude approximation or the steady-state assumption, and so these assumptions can themselves be investigated. In the case of the dependence on sweep rate, various proposed literature constraints need to be used with caution, because the exact validity is dependent on parameters such as rate constants, which are not known a priori. The simulations establish that a negative time constant should be a reliable indication of NGC mechanisms, which are measurable by dEIS even when the data is not KK transformable, though the parameters extracted may be inaccurate.

The concept of the rate determining step was discussed for a two-step adsorption mechanism. This simple example needs to be generalized by a detailed analysis, but it is evident that the concept of the rate determining step leads to simplifications in EIS spectra that can be easily understood qualitatively.

\section{Acknowledgments}

I thank the Natural Sciences and Engineering Council of Canada and the University of Victoria for financial support of this work.

\section{References}

1. R.L. Sacci and D.A. Harrington, ECS Trans., 19(20), 123 (2009).

2. D.A. Harrington and B.E. Conway, Electrochim. Acta, 32, 1703 (1987).

3. D.D. Macdonald, ECS Trans., 19(20), 55 (2009).

4. W.L. Underkofler and I. Shain, Anal. Chem., 37, 218 (1965)

5. A.M. Bond, R.J. O'Halloran, I. Ruzic and D.E. Smith, J. Electroanal. Chem., 90, 381 (1978).

6. D.A. Harrington, J. Electroanal. Chem., 355, 21 (1993). 
7. J.E. Garland, C.M. Petit and D. Roy, Electrochim. Acta, 49, 2623 (2004).

8. D.D. Macdonald and M. Urquidi-Macdonald, J. Electrochem. Soc., 132, 2316 (1985)

9. F. Seland, R. Tunold and D.A. Harrington, Electrochim. Acta, 51, 3827 (2006).

10. J. Sengoku, M. Naito, H. Okamoto, T. Ogita and S. Ichikawa, Chem. Phys. Lett., 525-526, 125 (2012). 